\title{
Fermi arcs tie the knot
}

\section{Cyclotron orbit knot and tunable-field quantum Hall effect}

Authors: Yi Zhang

arXiv:1905.02192

Phys. Rev. Research 1, 022005(R) (2019)

\section{Quantum Hall effect based on Weyl orbits in Cd3As2}

Authors: Cheng Zhang et al.

Nature, Vol 565, pg 331 (2019)

\section{Recommended with a Commentary by Ashvin Vishwanath, Harvard University}

Weyl fermions have been theoretically studied since 1929 when Hermann Weyl [1] wrote down his simplification of the massless Dirac equation. More recently, the realization of Weyl fermions in the electronic structure of crystalline solids opens up entirely new research questions which have no particle physics analog. It also holds the promise of experimental verification and perhaps even practical application. In particular the question of what happens at the edge of the Weyl 'universe' is most natural to pose in the context of solids, i.e. the surface states of Weyl semimetals. These surface states indeed turn out to be unusual and take the form of 'Fermi-arcs' [2], in which a closed two dimensional Fermi surface is shared between opposite faces of the crystal. The termination points of the Fermi arc line up with the Weyl points in the bulk Brillouin Zone, which appear in opposite chirality pairs. Indeed the peculiar nature of the Fermi arc surface states is reflected in their response to a magnetic field, and the formation of special cyclotron orbits which connect opposite surfaces of the sample. Experimental indications of these Weyl orbits were previously been reviewed in this Journal Club [3]. Each of the featured references give these ideas an unexpected 'twist' indicating that we have much more left to learn about Weyl fermions in crystals.

For two dimensional electrons or even for surface states on a three dimensional insulator, a vertical magnetic field lead to in-plane cyclotron orbits. However, for Fermi arc surface states, the electron needs to tunnel to the opposite surface to complete an orbit. Here, the gapless bulk of a Weyl semimetal must provide a conveyor belt - via chiral Landau levels - that relays the electrons between opposite surfaces as in Figure 1(a). The sense of the rotation of the surface states and the one-way propagation of chiral Landau levels between surfaces, set by the chirality of the Weyl fermion, must satisfy a matching condition to enable a consistent orbit. These are the 'Weyl Cyclotron Orbits' of Ref. [4, 5].

The featured theory Reference (1) asks the question - what happens when a more intricate connectivity between top and bottom surface Fermi arcs is present, as shown in Figure 1(b). 

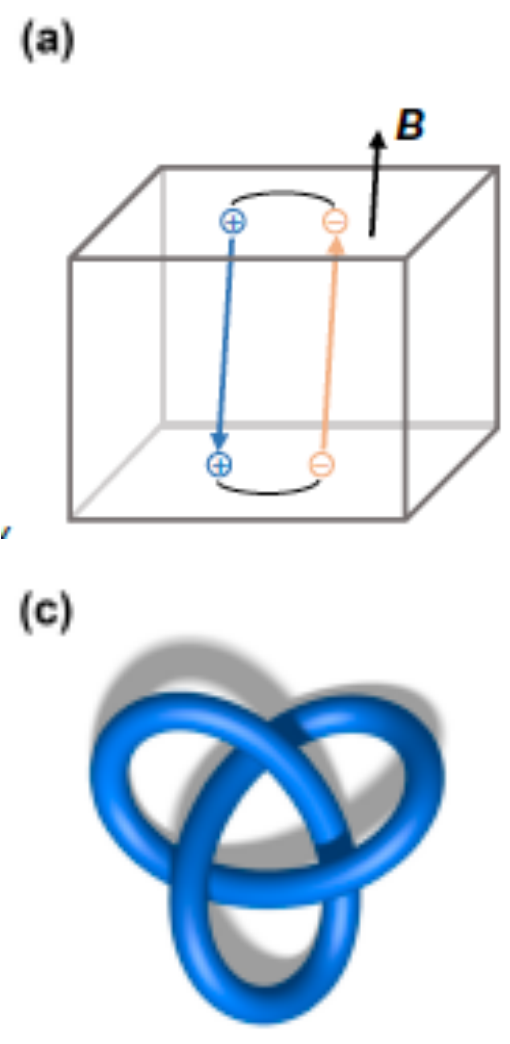
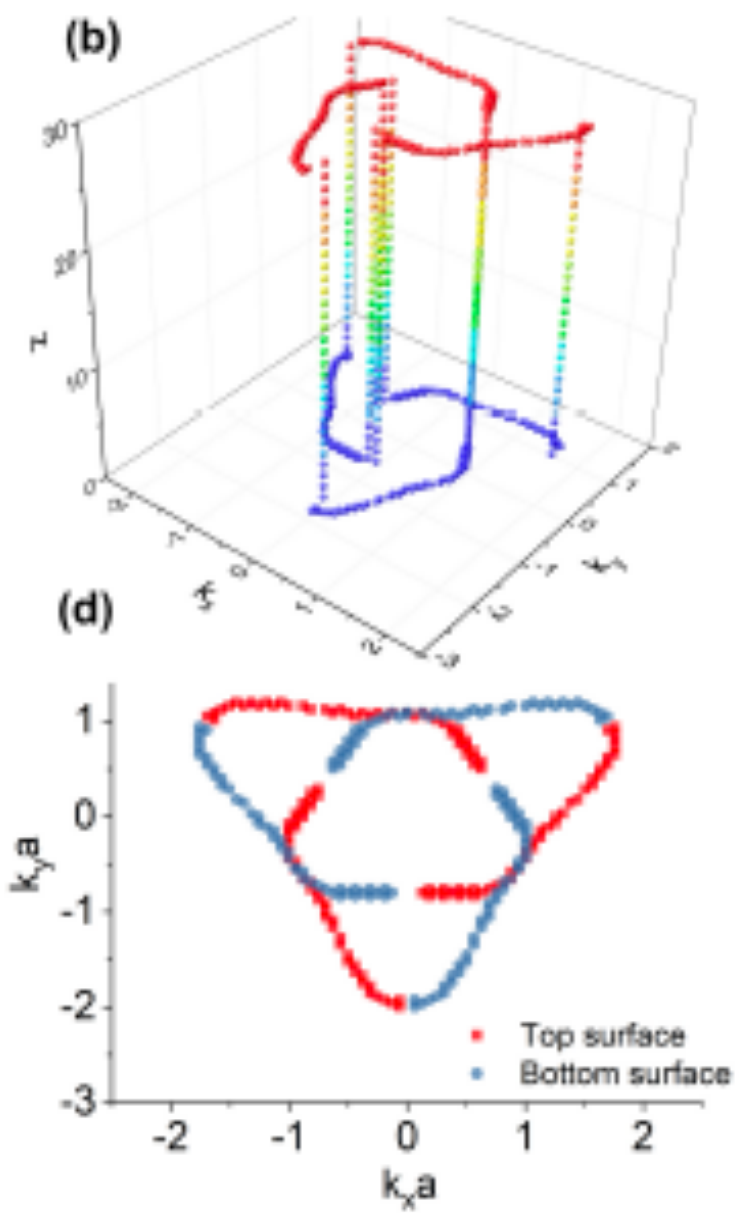

Figure 1: (a) Conventional Weyl cyclotron orbits connecting Fermi-arc surface states on opposite boundaries via bulk chiral Landau levels. (b) Fermi-arcs with twisted connectivity. The Weyl cyclotron orbits now sweep out a complex curve with the topology of a trefoil knot (figure (c) shows a trefoil knot). (d) The projection of the orbits as viewed from above with blue/red representing opposite surfaces. Note, the trajectories do not physically cross since they reside on opposite surfaces. (Taken from featured Reference 1) 
(a)

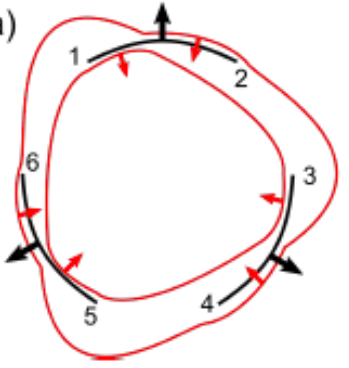

(c)

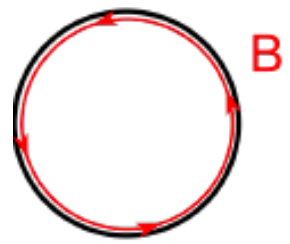

(b)

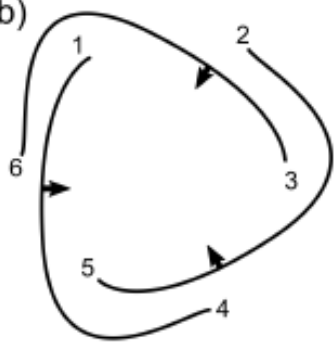

(d)

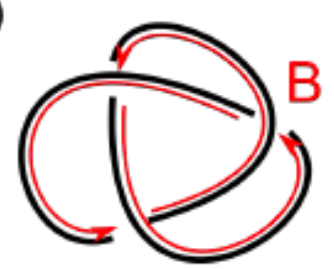

Figure 2: (a) Adding Fermi pockets and hybridizing with the usual Fermi arc surface states leads to (b) the reconnected configuration of Fermi arcs. (c) Magnetic field line along an unknotted Weyl orbit leads to no phase shift in contrast to (d) along a knotted Weyl orbit. (Taken from featured Reference 1)

Now, the Weyl cyclotron orbits must criss-cross the bulk multiple times and effectively trace out an orbit with the topology of a trefoil knot! The orbit is shown in the two dimensional momentum space parallel to the surface, but the real space orbit in the simply rotated by $90^{\circ}$ about the vertical axis. This immediately raises a couple of questions which are addressed in the reference. Q1) How do we obtain a pair of surfaces with that mutual pattern of Fermi-arcs ? It is shown in a model calculation that tuning surface properties, one can go from the 'conventional' Fermi arc scenario with matching arcs on opposite faces, to one with the knotted configuration. One can also think of transitioning between the two surface configurations by adding a metallic surface state that reconfigures the arcs (Figure 2a-2b)). And Q2) what is the physical consequence of this nontrivial topology of cyclotron orbit? It is argued that a strain engineered effective magnetic field along the orbit can lead to a phase shift in contrast to an unknotted Weyl orbit (Fig 2c-2d). It remains to be seen how a materials realization of this setup can be achieved and used to experimentally study this phenomena.

Finally in the second featured reference, a simpler scenario is studied experimentally, but which leads to its own surprise. A thin, wedge shaped topological semimetal $\mathrm{Cd}_{3} \mathrm{As}_{2}$ is studied in a perpendicular magnetic field. The variation in the height of the film is predicted to lead to chiral modes at certain well defined planes of constant height. The experimental signature in the form of a Hall signal in a Hall bar geometry with multiple contacts along the length of the bar (Figure 4) is found to be consistent with these expectations (an explanation based on the finite size quantization of purely bulk states had to be ruled out through explicit calculations). These results point to a rich set of phenomena that combine the old problem 
a)

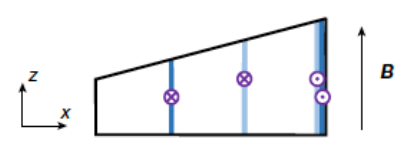

c)

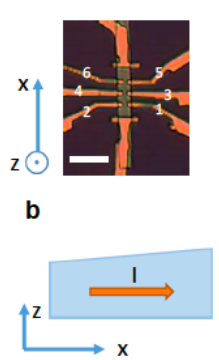

b)

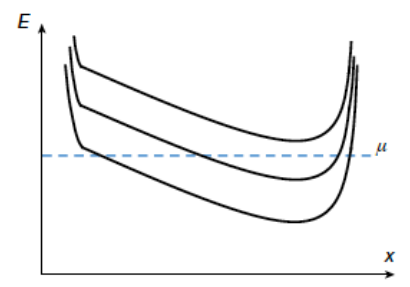

d)

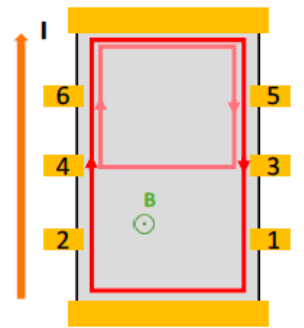

Figure 3: (a) Wedge shaped sample with chiral edge states delocalized through the bulk in the $y-z$ plane, but at a fixed ' $x$ ' as predicted by (b) Landau level diagram. (c, d) Experimental setup and expected configuration of chiral modes (Taken from featured Reference 2)

of magnetic oscillations and quantum Hall effect, with the three dimensional topology of Weyl fermions. Future progress in this theory-experiment partnership will require new and ideal materials with greater tunability, a better understanding of finite size effects and of course creative new ideas like those contained in the featured references.

\section{References}

[1] H. Weyl, "Electron and gravitation", Z. Phys. 56 (1929) 330-352 [English translation: Surveys High Energ. Phys. 5 (1986) 261-267].

[2] Wan, X. et al., "Topological semimetal and Fermi-arc surface states in the electronic structure of pyrochlore iridates". Phys. Rev. B 83, 205101 (2011).

[3] "Emergent technology based on Fermi-arcs?" Journal Club for Condensed Matter Physics by Luis Balicas, November 30, 2016. DOI: 10.36471JCCM_November_2016_01

[4] A. Potter et al. "Quantum oscillations from surface Fermi arcs in Weyl and Dirac semimetals." Nat. Commun. 5, 5161 (2014).Yi Zhang et al. "Quantum oscillations from generic surface Fermi arcs and bulk chiral modes in Weyl semimetals." Sci. Rep. 6, 23741 (2016). Y. Baum et al. "Current at a Distance and Resonant Transparency in Weyl Semimetals." Phys. Rev. X 5, 041046 (2015).

[5] Moll, P. J. et al. "Transport evidence for Fermi-arc-mediated chirality transfer in the Dirac semimetal $\mathrm{Cd}_{3} \mathrm{As}_{2}$." Nature 535, 266-270 (2016). 